\title{
A novel inversion technique for imaging thrombus volume in microchannels fusing optical and impedance data
}

\author{
A. Affanni ${ }^{\sharp}$, G. Chiorboli ${ }^{\natural}$, L. Codecasa ${ }^{\star}$, M.R. Cozzi ${ }^{b}$, L. De Marco ${ }^{b}$, M. Mazzucato ${ }^{b}$, \\ C. Morandi ${ }^{\natural}$, R. Specogna ${ }^{\sharp}$, M. Tartagni*, F. Trevisan ${ }^{\sharp}$ \\ \#Dep. of Electrical, Mechanics and Management Engineering, Università di Udine, Via delle Scienze 206, 33100 Udine, Italy \\ ${ }^{\natural}$ Dep. of Information Engineering, Università di Parma, Viale G.P. Usberti 181/A, 43100 Parma, Italy \\ •Dep. of Electronics and Information Engineering, Politecnico di Milano, via Ponzio 34/5, 20133 Milano, Italy \\ ${ }^{b}$ National Cancer Institute CRO-IRCCS, Via Franco Gallini 2, 33081 Aviano (PN), Italy \\ *Dep. of Electrical and Information Engineering, Università di Bologna, Viale del Risorgimento 2, 40136 Bologna, Italy \\ ruben.specogna@uniud.it
}

\begin{abstract}
The aim of this paper is to present a novel inversion technique to measure the volume of thrombus induced under blood flow conditions in a lab-on-a-chip device. The device is composed by a microscope slide where parallel gold electrodes are sputtered and by a PDMS microchannel placed on the top of the slide. A thrombogenic substance is placed on the slide in such a way that hemostasis is induced when whole blood flows in the microchannel. The novel idea behind the inversion technique is to fuse optical and electrical impedance data to obtain a quasi real-time reconstruction of thrombus volume. This is not possible with the present state of the art optical imaging based on confocal microscopy that provides the thrombus volume estimation only at the end of the thrombus formation.
\end{abstract}

Index Terms-Biomedical engineering, bioimpedance, biomedical imaging, biosensors

\section{INTRODUCTION}

In the perspective of a point-of-care prevention screening system, there is an increasing interest in in vitro measurement of thrombus formation under flow conditions to profile the thrombotic process in humans. Current available technologies capture the kinetics of thrombus formation in collagen coated artificial capillary or flow chamber by means of a video imaging technique and image processing which include a microscopy device either inverted or confocal. For the kinematic imaging of thrombus formation a fluorescent labeling for platelets is used. In the inverted microscope the quantification of thrombus formation is performed from a digital bidimensional data image of a whole blood sample based on pixel luminance. The 3D size of the thrombus is therefore mathematically extrapolated only from the pixel luminance and may not reflect its actual size. This problem can be overcame by the use of a confocal microscope, but the size of the instrument and its costs are against its usage in a bedside clinical setting. Moreover, confocal microscopy provides the thrombus volume only at the end of the process.

A preliminary electrical characterization of thrombus formation in microchannels under blood flow conditions by means of

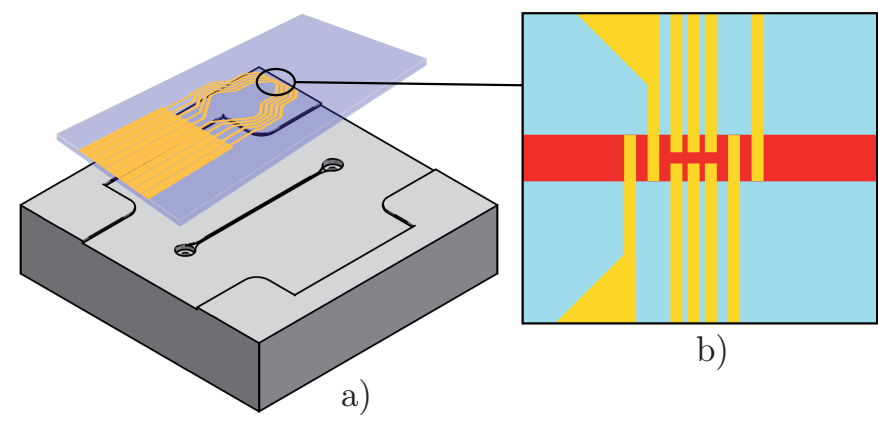

Figure 1: (a) Assembly of the sensor composed of PDMS microchannel and microelectrodes sputtered on a microscope slide; (b) zoom on microelectrodes.

Electrical Impedance Spectroscopy (EIS) has been presented in [1] using an impedance meter and a low cost sensor based on copper PCB technology and improved in [2] using a pair of gold microwires.

In this paper, we present a novel and cheap system that should provide information on the individual thrombotic profile. This is of fundamental importance for the identification of the best pharmacological strategy to adopt for each patient. This technique evaluates the volume of thrombus during its formation, by monitoring the global electrical impedance of blood flowing on a thrombogenic surface together with a 2D optical image of pixel luminance.

\section{LAB-ON-A-CHIP DEVICE}

In this paper, to detect thrombus formation, we designed a lab-on-a-chip sensor consisting of multiple pairs of parallel gold electrodes, sputtered only on the surface of the slide closing the microchannel and transversally with respect to the blood flow direction, see Fig. 1.

The fluidic connections and an open microchannel without 
the upper wall are molded on the PDMS substrate; the channel dimensions are $400 \mu \mathrm{m}$ in width, $250 \mu \mathrm{m}$ in depth and $25 \mathrm{~mm}$ in length. The upper wall of the microchannel is formed by a slide with $200 \mathrm{~nm}$ thick sputtered gold electrodes, arranged as shown in Fig. 1b, where type I collagen is coated.

\section{NOVEL INVERSION TECHNIQUE}

In order to reconstruct the thrombus geometry and volume by means of a novel inversion methodology based on fusing optical and impedance data, a first electrode arrangement is used consisting of a couple of electrodes whose length extends over the entire microchannel width and the electrode width is $100 \mu \mathrm{m}$, the electrodes gap is $100 \mu \mathrm{m}$; referring to Fig. $1 \mathrm{~b}$ this arrangement represents the two couples posed on the left and on the right of the sketch.

With such a sensor, at a time instant and at a specific frequency, the acquired impedance data are post-processed and compared with respect to the corresponding impedance computed by means of an electromagnetic simulation. The geometry of the thrombus is represented by a solid model deduced from the 2D optical image of pixel luminance. For each pixel, the height of the thrombus with respect to surface of the slide, is assumed to be proportional to the luminance according to a unique scale factor for all the pixels. In order to determine such a scale factor, a number of 3D steadystate current conduction analyses of the overall system are performed by modifying the scale factor until the computed impedance matches the measured one; the thrombus volume is then easily evaluated by the thrombus solid model.

The electrical parameters of blood flowing in microchannels at high shear rates that have been used in the simulations can be found in [4], [5], whereas the thrombus can be considered as a perfect insulator below a few hundreds of $\mathrm{kHz}$.

The 3D steady-state current conduction analysis tool expressly developed for this application is based on the Discrete Geometric Approach developed by the authors [3], [4]. More details on the efficient forward solver and all the simulation parameters will be included in the full paper.

\section{Experimental Results}

The impedance measurements were performed connecting the slide to the Agilent E4980A precision LCR meter and automatically acquiring the impedance data measured with a two-wire technique by a software control panel. In the final device we are planning to implement impedance detection using an integrated circuit device.

Blood flow rate was kept constant in order to obtain a shear rate of $3000 \mathrm{~s}^{-1}$ in a $37{ }^{\circ} \mathrm{C}$ controlled temperature perfusion chamber and experiments were performed for a standard duration of five minutes.

The continuous line plotted in Fig. 2 shows the impedance magnitude at $150 \mathrm{kHz}$ frequency, whereas the markers represent the thrombus volume reconstructed in 12 frames. Figure 3 shows the $2 \mathrm{~d}$ image captured with the microscope and the thrombus geometry reconstructed by the proposed method relative to the time frame highlighted in Fig. 2.

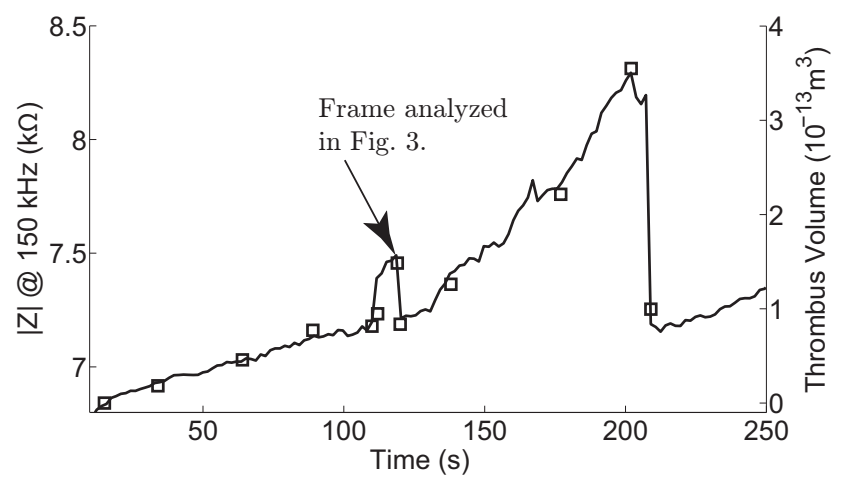

Figure 2: (-): Behavior of the impedance magnitude versus time at $150 \mathrm{kHz}$ when aggregation is taking place; ( $\mathbf{\square})$ : reconstructed thrombus volume.

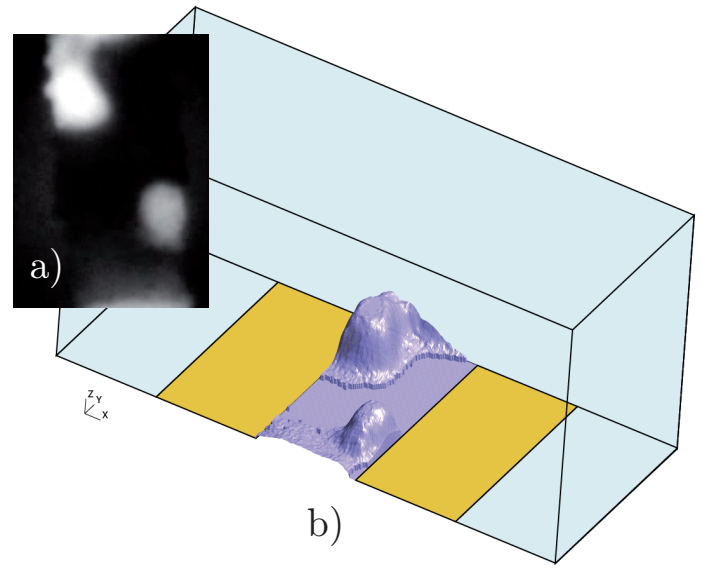

Figure 3: a) Microscope image and b) reconstructed thrombus geometry at the time frame highlighted in Fig. 2.

\section{Acknowledgments}

This work is Partially supported by the Italian Ministry of Education, University and Research (MIUR) project PRIN 2009LTRYRE.

\section{REFERENCES}

[1] A. Affanni, R. Specogna, F. Trevisan "Measurement Bench for Impedance Tomography during Hemostasis Process in whole Blood", IEEE International Symposium on on Medical Measurements and Applications (MeMeA 2011) Proceedings, Bari (Italy), 30-31 May 2011, pp. 80-83.

[2] A. Affanni, R. Specogna, F. Trevisan "Electrical Impedance Spectroscopy on flowing blood to predict white thrombus formation in artificial microchannels", IEEE International Instrumentation and Measurement Technology Conference (I2MTC2012) Proceedings, Graz (Austria), 13-16 May 2012, pp. 1477-1480.

[3] P. Bettini, R. Specogna, and F. Trevisan, "Electroquasistatic analysis of the gas insulated line for the ITER neutral beam injector, IEEE Trans. Magn., vol. 45, pp. 996999, 2009.

[4] A. Affanni, R. Specogna, F. Trevisan "A Discrete Geometric Approach to Cell Membrane and Electrode Contact Impedance Modeling", IEEE Trans. Biomed. Eng., vol. 59, pp. 2619-2627, 2012.

[5] A. E. Hoetink, Th. J. C. Faes, K. R. Visser, and R. M. Heethaar, "On the Flow Dependency of the Electrical Conductivity of Blood", IEEE Trans. Biomed. Eng., vol. 51, pp. 1251-1261, 2004. 